\title{
Quantitative Immunochromatographic Strip Biosensor for the Detection of Carcinoembryonic Antigen Tumor Biomarker in Human Plasma
}

\author{
Qingxiang Zeng, ${ }^{\text {a, b }}$ Xun Mao, ${ }^{\text {b Hui Xu }},{ }^{\text {a, b }}$ Shengfu Wang, ${ }^{\text {a } b^{*}}$ Guodong Liu ${ }^{\text {b* }}$
}

${ }^{a}$ College of Chemistry and Chemical Engineering, Hubei University, Wuhan 430062, P. R. China

${ }^{\mathrm{b}}$ Department of Chemistry and Molecular Biology, North Dakota State University, Fargo, ND, 58105

*Corresponding Authors:

G. Liu and S. Wang

Phone: 701-231-8697

Fax: 701-231-8831

E-mail address: guodong.liu@ndsu.edu (G. Liu), wangsf@hubu.edu.cn (S. Wang)

Received: 15 December 2008; | Revised: 16 January 2008; | Accepted: 18 January 2009

\begin{abstract}
Combining the unique optical properties of gold nanoparticle (Au-NP) and the antibody-antigen specific molecular recognition, a quantitative immunochromatographic strip biosensor (QISB) in connection with a portable strip reader was developed for the detection of carcinoembryonic antigen (CEA) tumor biomarker in human plasma. The test is based on the principle of sandwich-type immunreaction on the lateral flow test strip. The polyclonal antibody $(\mathrm{Ab})$ and $\mathrm{Au}-\mathrm{NP}$ labeled monoclonal $\mathrm{Ab}$ are employed to identify CEA specifically. The quantitative detection of CEA was realized by recording the optical intensities of the produced red bands in the strip biosensor with the portable strip reader. The biosensor fabrication and assay parameters (e.g. membrane sources, the concentration of polyclonal $\mathrm{Ab}$ in the test zone, the amount of $\mathrm{Au}$ NP-anti-CEA conjugates and the components of running buffer) that govern the sensitivity and reproducibility of the biosensor were optimized. Under optimal conditions, the response of the QISB is linear in the range of $10-200 \mathrm{ng} \mathrm{mL}^{-1}$ with a detection limit of $5 \mathrm{ng} \mathrm{mL}^{-1}(\mathrm{~S} / \mathrm{N}=3)$ in association with a $10-\mathrm{min}$ assay time. The biosensor has also been successfully applied for the detection of CEA in human serum sample. The QISB thus provides a rapid, sensitive, low cost clinical diagnosis tool for the detection of protein biomarkers in biological samples.
\end{abstract}

Keywords: CEA, Tumor biomarker, Gold nanoparticle, Immunochromatographic, Quantitative, Biosensor

\section{Introduction}

Carcinoembryonic antigen (CEA), a glycoprotein first described in 1965 by Gold and
Freedman [1], is one of the most widely used tumor makers, and is used in the clinical diagnosis of breast cancer [2-4], colon cancer [5-7], lung cancer $[8,9]$ and ovarian carcinoma [10]. CEA 
level in serum is also related to the state of the tumor, so it can be used as a marker to directly evaluate curative effects, recrudescence, and metastasis. The techniques usually used for quantitative determination of tumor markers are immunological methods, which have become the predominant analytical techniques in the fields of clinical diagnoses and biochemical studies. A number of immunoassay methods including radioimmunoassay [11- 13], fluoroimmunoassay [14, 15], chemiluminescence immunoassay [16] and enzyme-linked immunosorbent assays (ELISAs) [17] have been employed in detecting serum CEA for clinical diagnosis. Although these conventional strategies provide accurate, sensitive detection of CEA, there are still some inconveniences exist, such as the utilization of radioactive substances, time-consuming sample purification, incubation, washing steps before analysis and enzymatic reactions, technical expertise as well as the specialized equipment.

The immunosensor is an alternative tool to replace the traditional immunoassay. A number of immunosensors in connection with various transducers have been reported for the detection of CEA [18-22]. Although most of the reported CEA immunosensors have simplified the operations, shortened the assay time, and provided a good sensitivity, applications of the developed CEA immunosensors stay in the laboratorydevelopment level and have not been widely used for clinical diagnosis. Immunochromatographic strip tests (ISTs) are simple, rapid, in-field and cheap assays. The principle of IST relies on the migration of test samples and antibody-gold nanoparticle (Ab-Au-NP) conjugates along membrane strips on which the binding interactions take place. The results of IST could be examined visually, thus providing fast and simple qualititative detection without the need of skilled personnel [24-28]. However the ISTs are used for qualititative or semi-quantitative detection, and fail to offer the quantitative information of the interested target in the samples. Much effort has been made to the development of quantitative IST. Kim and his colleagues have reported the fluorescence IST for quantifying analytes [29]. However, Fluorescence ISTs were often complicated by the requirement of an elaborate excitation and detection scheme and by the broad emission bands. Liu and co-workers developed a disposable electrochemical immunosensor diagnosis device based on nanoparticle probe and ITS for quantifying $\operatorname{IgG}$ and PSA [30, 31]. Although IST coupled with the portable electrochemical detector shows promise for pointof-care applications [30-32], the additional dissolution step (dissolving the captured nanoparticles in the test zone) and the use of strong acids and toxic mercury (for stripping voltammetric measurements) prevent its wide applications.

In this paper, we present a simple and convenient diagnosis tool based on the lateral flow technology and a portable strip reader for rapid and quantitative detection of CEA in human plasma. The biosensor overcomes the disadvantages of the reported CEA immunosensors and qualitative CEA test strips, thus provides a rapid, sensitive, low cost clinical diagnosis tool for the detection of protein biomarkers in biological samples.

\section{Experimental}

Apparatus. Airjet AJQ 3000 dispenser, Biojet BJQ 3000 dispenser, Clamshell Laminator and the Guillotine cutting module CM 4000 were from Biodot LTD (Irvine, CA) and used for the preparation of CEA strip biosensor. A portable strip reader DT1030 was purchased from Shanghai Goldbio Tech. Co., LTD (Shanghai, China).

Reagents. CEA, polyclonal CEA antibody (poly-anti-CEA), monoclonal CEA antibody (antiCEA, clone \#:M111147) were purchased from Fitzgerald (USA); Goat anti-mouse $\mathrm{IgG}$, rabbit IgG and human IgM were purchased from Thermo scientific; Human plasma samples were purchased from Golden West Biologicals (Temecula, CA). $\mathrm{Na}_{3} \mathrm{PO}_{4} \bullet 12 \mathrm{H}_{2} \mathrm{O}, \quad \mathrm{HAuCl}_{4}$, trisodium citrate, sucrose, Tween 20 , sodium chloride-sodium citrate (SSC) buffer ( $\mathrm{pH} 7.0$ ), phosphate buffer saline (PBS, PH 7.4, $0.01 \mathrm{M}$ ), bovine serum albumin (BSA), human serum albumin (HAS) were purchased from Sigma-Aldrich. Glass fibers (GFCP000800), cellulose fiber sample pads (CFSP001700), laminated cards (HF000MC100) and nitrocellulose membranes (HFB18004 and HFB 24004) were purchased from Millipore 
(Billerica, MA). All chemicals used in this study were analytical reagent grade. All other solutions were prepared with ultrapure $(>18 \mathrm{M} \Omega$ ) water from a Millipore Milli-Q water purification system (Billerica, MA).

\section{Preparation of Gold Nanoparticles (Au-NP) and Au-NP-anti-CEA Conjugates.}

Gold nanoparticles with average diameter 20 $\mathrm{nm} \pm 3.5 \mathrm{~nm}$ used in this work were prepared according to the citrate reduction of $\mathrm{HAuCl}_{4}[33$, 34]. All glassware used in this preparation was thoroughly cleaned in aqua regia (three parts $\mathrm{HCl}$, one part $\mathrm{HNO}_{3}$ ), rinsed with double distilled $\mathrm{H}_{2} \mathrm{O}$, and oven-dried prior to use. A $250 \mathrm{~mL}$ aqueous solution of $0.01 \% \mathrm{HAuCl}_{4}$ was added to a $500 \mathrm{~mL}$ round-bottom flask and heated to boiling with vigorously stirring, and then added $4.5 \mathrm{~mL}$ of $1 \%$ trisodium citrate to this solution quickly. The solution turned deep blue within $20 \mathrm{~s}$ and the finally changed to wine-red $60 \mathrm{~s}$ later. Boiling was continued for an additional 10 minutes, the heating source was removed, and the colloid was stirred for another $15 \mathrm{~min}$. The colloids solution were stored in dark bottles at $4{ }^{\circ} \mathrm{C}$ and were used to prepare Au-NP-anti-CEA conjugate.

The conjugation was carried out by adding 50 $\mu \mathrm{L}$ of $1 \mathrm{mg} / \mathrm{mL}$ anti-CEA monoclonal $\mathrm{Ab}$ to $1 \mathrm{~mL}$ of 5-fold concentrated Au-NP solution ( $\mathrm{pH} 8.2$ ) followed by incubation at room temperature with periodic gentle mixing for $1 \mathrm{hr}$. Then certain volume of $10 \%$ BSA was slowly added to the mixture solution to a final concentration of $1 \%$. After gentle stirring for 30 minutes, the solution was centrifuged at $13,000 \times g$ for $15 \mathrm{~min}$. Two phases can be obtained: a clear to pink supernatant of unbound antibodies and a dark red, loosely packed sediment of the Au-NP-anti-CEA conjugates. The supernatant was discarded and the soft sediment of Au-NP-anti-CEA conjugates was rinsed by resuspending in $1 \mathrm{ml}$ of PBS-BSA and collected after a second centrifugation at $13000 \times g$ for $15 \mathrm{~min}$. Finally, the conjugate was resuspended in $1 \mathrm{~mL}$ of buffer containing $20 \mathrm{mM}$ sodium phosphate, $0.25 \%$ Tween-20, $10 \%$ sucrose and $5 \%$ BSA).

\section{Preparation of CEA Strip Biosensors}

A schematic diagram of the CEA strip biosensor is shown in Figure 1 (A). Briefly, the biosensor consists of four components: sample application pad, Au-NP-anti-CEA conjugate pad, nitrocellulose membrane and absorbent pad. The sample application pad $(17 \mathrm{~mm} \times 30 \mathrm{~cm})$ was made from cellulose fiber (CFSP001700, Millipore) and saturated with a buffer ( $\mathrm{pH} 8.0)$ containing $0.25 \%$ Triton X-100, $0.05 \mathrm{M}$ Tris- $\mathrm{HCl}$ and $0.15 \mathrm{mM} \mathrm{NaCl}$. Then it was dried and stored in desiccators at room temperature. The conjugate pad $(8 \mathrm{~mm} \times 30 \mathrm{~cm})$ was prepared by dispensing a desired volume of Au-NP-anti-CEA conjugate solution onto the glass fiber with the dispenser Airjet AJQ 3000, and then drying it in oven at 35 ${ }^{\circ} \mathrm{C}$ and stored in desiccators at $4{ }^{\circ} \mathrm{C}$. Test zone and control zone at the nitrocellulose membrane (25 $\mathrm{mm} \times 30 \mathrm{~mm}$ ) was prepared by dispensing polyanti-CEA and goat anti-mouse IgG with Biojet BJQ 3000, respectively. The distance between two zones is around $0.2 \mathrm{~cm}$. The membrane was dried at room temperature for $1 \mathrm{~h}$ and stored at $4{ }^{\circ} \mathrm{C}$. Finally, all of the parts were assembled on a plastic adhesive backing layer (typically an inert plastic, e.g., polyester) using the Clamshell Laminator (BioDot, CA, USA). Each part overlapped $2 \mathrm{~mm}$ to ensure the solution migrating through the strip during the assay. QISBs with a $4.7 \mathrm{~mm}$ width were cut by using the Guillotin cutting module CM 4000.

\section{Sample assay Procedure}

During optimizing the experimental parameters of the biosensor, $120 \mu \mathrm{L}$ of sample solution containing a desired concentration of CEA (prepared in PBS $+1 \%$ BSA buffer) was added onto the sample application pad. After waiting for $4 \mathrm{~min}, 50 \mu \mathrm{L}$ of buffer (PBS $+1 \%$ BSA) was applied to the strip. Both the test zone and control zone were visualized within $10 \mathrm{~min}$. For quantitative measurements, the optical intensity of the two red bands was recorded in the portable "strip reader" (Figure $1 \mathrm{~B}$ ) with "GoldBio strip reader" software. In the case of detecting CEA in plasma sample, $120 \mu \mathrm{L}$ of human plasma spiked with certain concentration of CEA was applied to the strip, and then the strip was washed with PBS $+1 \%$ BSA as described above. After 10 min, the optical response of strip was recorded by strip reader. 


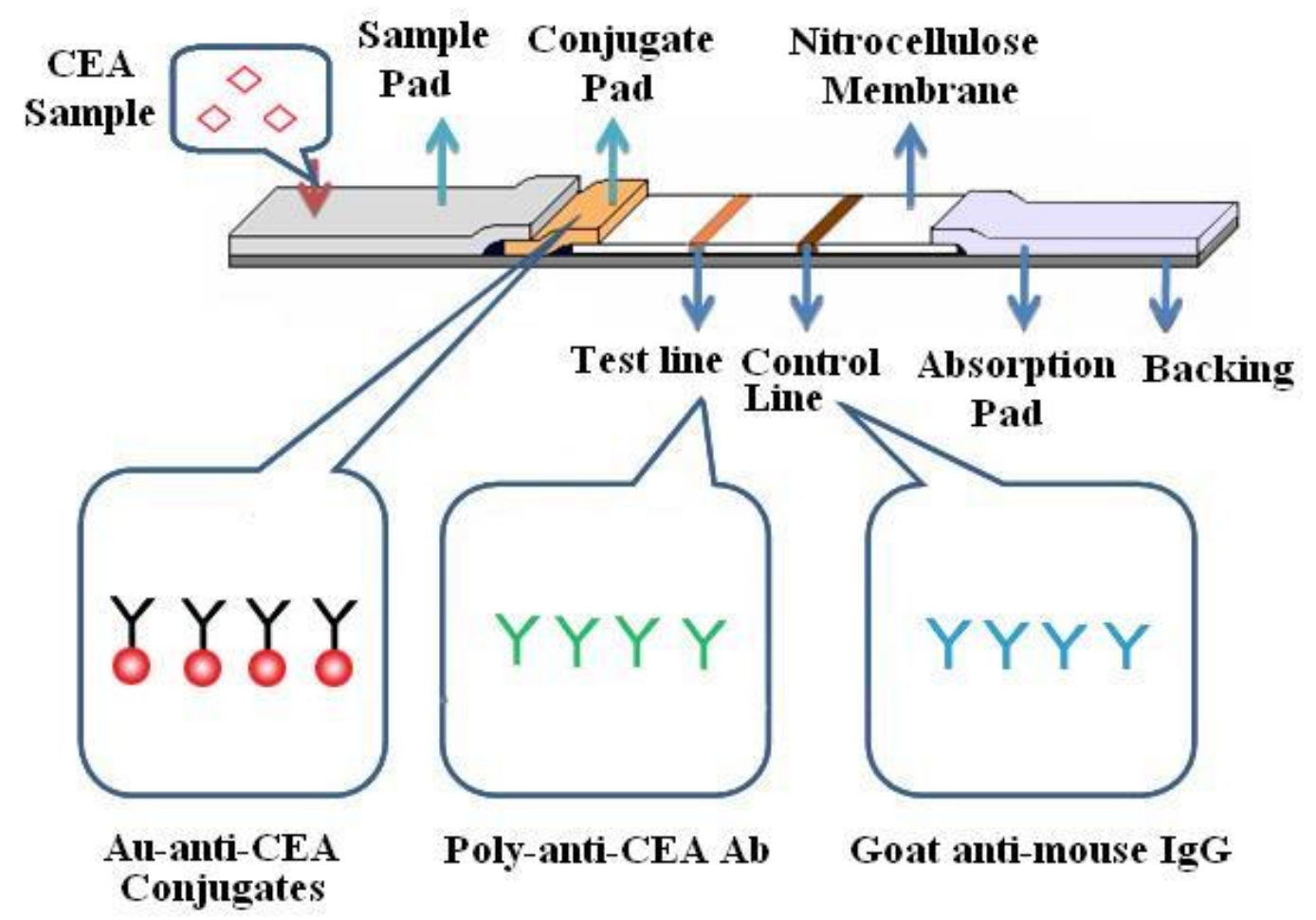

(A)

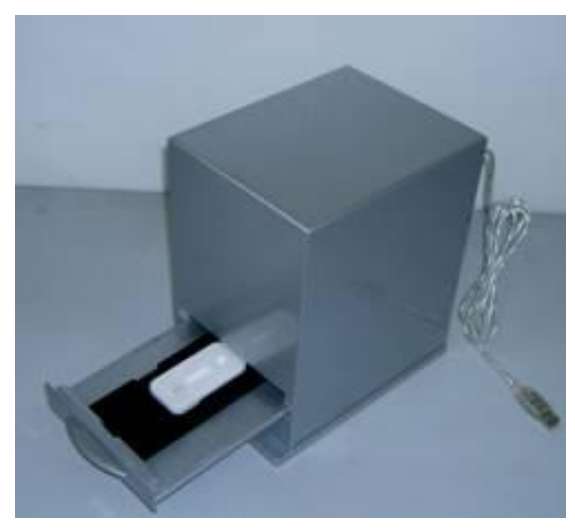

(B)

Figure 1. (A) Schematic illustration of CEA strip biosensor; (B) Photo image of portable strip reader.

\section{Results and Discussions}

Principle of CEA Measurement in the Strip Biosensor. The principle of the biosensor is based on sandwich-type immunoreactions in the lateral flow test strip as illustrated in Figure 2. Ploy-anti$\mathrm{CEA} \mathrm{Ab}$ and goat anti-mouse $\operatorname{IgG}$ solutions were dispensed on the different locations of the nitrocellulose membrane to form a test zone and a control zone, respectively. The Au-NPs were used as tags to label monoclonal anti-CEA Ab, the resulting Au-NP-anti-CEA conjugates were dispensed onto the glass fiber as the conjugate pad. In a typical assay, a sample solution containing a desired concentration of CEA was applied to the sample application pad. 
A. Sampling

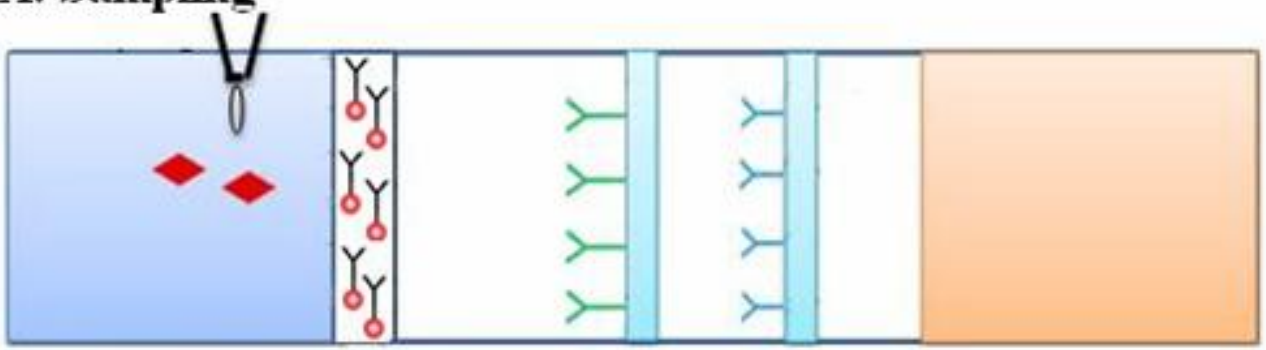

\section{B. Immumoreaction and Migration}

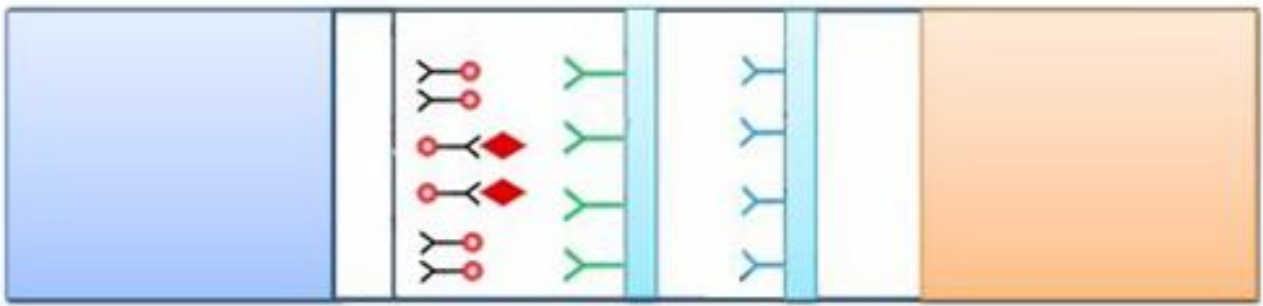

\section{Capture of CEA by Poly-anti-CEA Ab on test line}

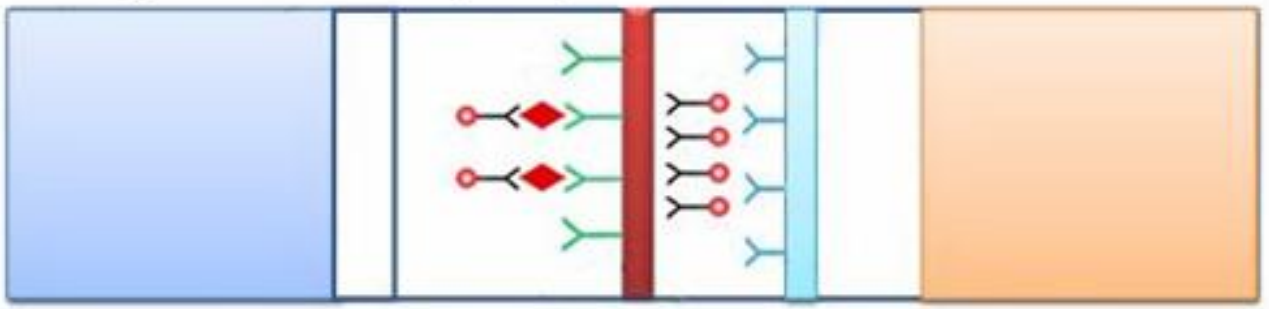

D. Capturing excess Au-anti-CEA conjugates on control line

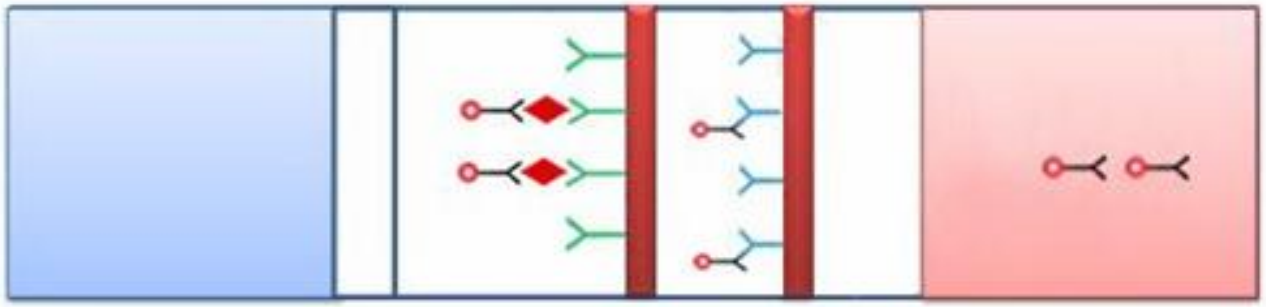

\section{E. Record the intensity of the formed red line with strip reader}

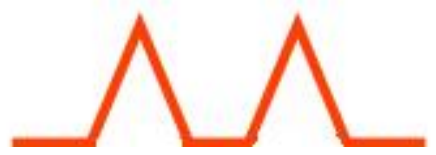

Figure 2. Principle of quantitative detection of CEA in the strip biosensor.

The solution migrates along the strip by capillary force and rehydrates the Au-NP-antiCEA in the conjugate pad. Then the immunoreactions between CEA and Au-NP-antiCEA conjugates occurred and the formed Au-NPanti-CEA-CEA complexes continue to migrate along the strip. When reached the test zone, the complexes were then captured by the poly-anti$\mathrm{CEA} \mathrm{Ab}$ immobilized on the test zone via the secondary immunoreactions between the polyanti-CEA Ab and CEA. A characteristic red band could be observed because of the accumulation of Au-NPs on the test zone. The capillary action caused liquid sample to migrate further. Once the 
solution passed through the control zone, the excess Au-NP-anti-CEA conjugates were captured on the control zone via the binding between the goat anti-mouse IgG antibody (pre-immobilized on the control zone) and the anti-CEA Ab, thus forming a second red band. In the absence of CEA, only the red band is observed in the control zone and no red band is observed in the test zone. In this case, the red band in the control zone (control line) shows that the biosensor is working properly. Qualitative analysis is simply performed by observing the color change of the test zone, and quantitative analysis is realized by reading the optical intensities of the red bands with the portable strip reader (also see Figure 1(B)). The peak area is proportional to the amount of the captured Au-NPs in the test zone, which is proportional to the concentration of CEA in the sample solution.

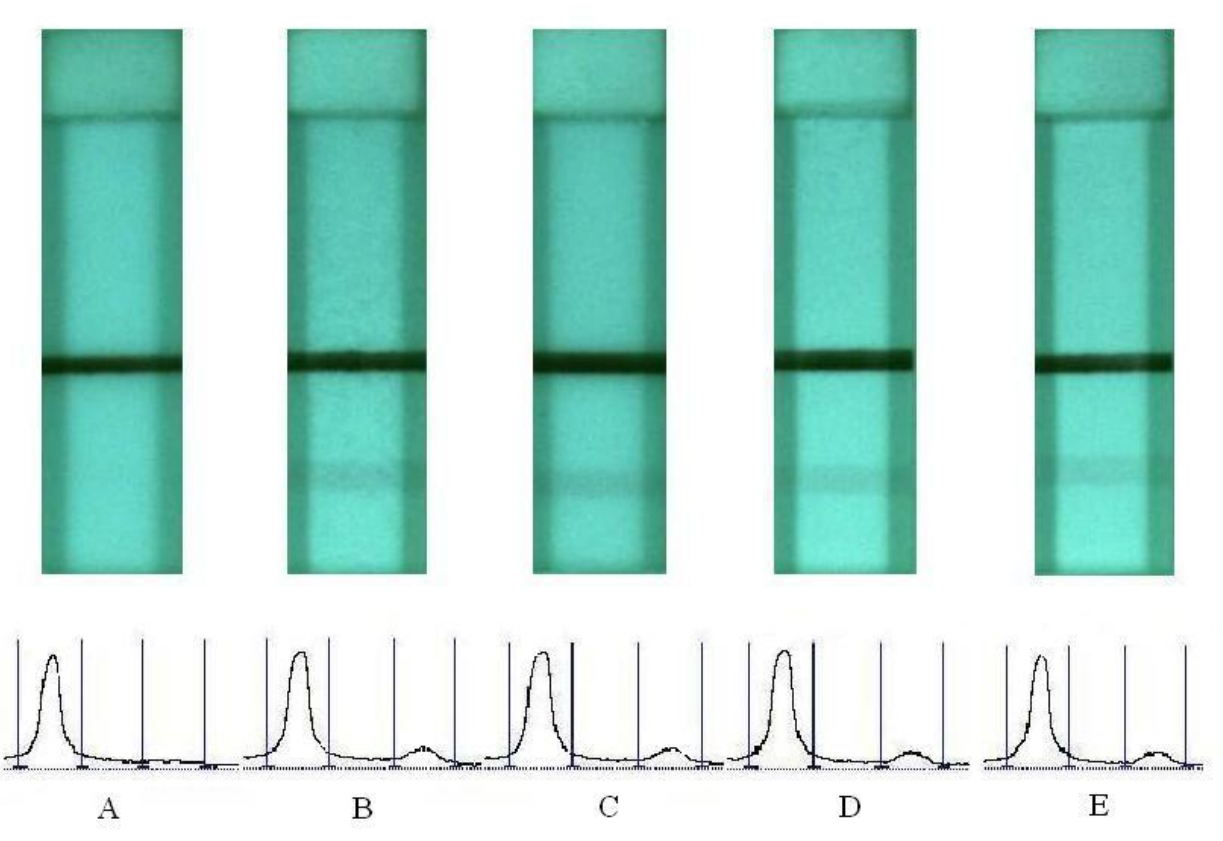

Figure 3. Typical photo images and recorded response signals of the test strip with a portable strip reader after applying the sample solutions. (A) $0 \mathrm{ng} \mathrm{mL}^{-1} \mathrm{CEA}$, (B) $100 \mathrm{ng} \mathrm{mL}^{-1} \mathrm{CEA}$, (C) $100 \mathrm{ng} \mathrm{mL} \mathrm{CEA}^{-1} 1 \mu \mathrm{g}$ $\mathrm{mL}^{-1}$ rabbit IgG, (D) $100 \mathrm{ng} \mathrm{mL} \mathrm{L}^{-1} \mathrm{CEA}+1 \mu \mathrm{g} \mathrm{mL} \mathrm{m}^{-1}$ human IgM, (E) $100 \mathrm{ng} \mathrm{mL} \mathrm{mL}^{-1} \mathrm{CEA}+1 \mu \mathrm{g} \mathrm{mL}^{-1} \mathrm{HSA}_{\text {. }}$ All sample solutions were prepared with $0.01 \mathrm{M}$ PBS $+1 \%$ BSA buffer; 0.01M PBS $+1 \%$ BSA solution as running buffer; 4 times of dispersing of Au-anti-CEA conjugates on conjugates pad; the quantity of polyanti-CEA Ab used for the preparation of test line is 6 times dilution of its stock concentration.

Figure 3 displays the typical photo images and corresponding optical responses of $0 \mathrm{ng} / \mathrm{mL}$ CEA (A, control), $100 \mathrm{ng} / \mathrm{mL}$ CEA (B), 100 $\mathrm{ng} / \mathrm{mL} \mathrm{CEA}+1 \mu \mathrm{g} / \mathrm{mL}$ rabbit $\mathrm{IgG}(\mathrm{C}), 100 \mathrm{ng} / \mathrm{mL}$ $\mathrm{CEA}+1 \mu \mathrm{g} / \mathrm{mL}$ human IgM (D), $100 \mathrm{ng} / \mathrm{mL}$ CEA $+1 \mu \mathrm{g} / \mathrm{mL}$ HSA $(\mathrm{E})$. There were two visible bands appeared in the presence of $\mathrm{CEA}(\mathrm{B})$, and only one band (A) could be seen in the absence of CEA. The excess of nonspecific proteins, such as $\mathrm{IgG}$, IgM and HAS do not interfere the response of the biosensor $(C, D, E)$. The optical intensities of the bands are proportional to the amount of Au-NPs, then the concentration of CEA in the sample solutions.

\section{Optimization of Parameters.}

In the current study, sandwich-type immunoreactions were performed on the lateral flow test strip biosensor, the immunoreaction time, which depends on the migration time of buffer in the nitrocellulose membrane, plays important role for the sensitivity of the biosensor. Two 
nitrocellulose membranes including HFB 18004 and HFB 24004 (Millipore) were choose to prepare the strip biosensor. According to the instruction of manufacture, the migration times of the buffer in HFB 18004 and HFB24004 membranes are $3 \mathrm{~min}$ and $4 \mathrm{~min}$, respectively. Figure 4 (A) presents the signal to noise $(\mathrm{S} / \mathrm{N})$ ratio of the biosensor prepared by the above nitrocellulose membranes. One can see the $\mathrm{S} / \mathrm{N}$ ratio with the HFB 18004 nitrocellulose membrane is higher than that with the HFB 24004. The assay time with HFB 18004 is around $10 \mathrm{~min}$. So the HFB 18004 nitrocellulose membrane was used to prepare the biosensor.
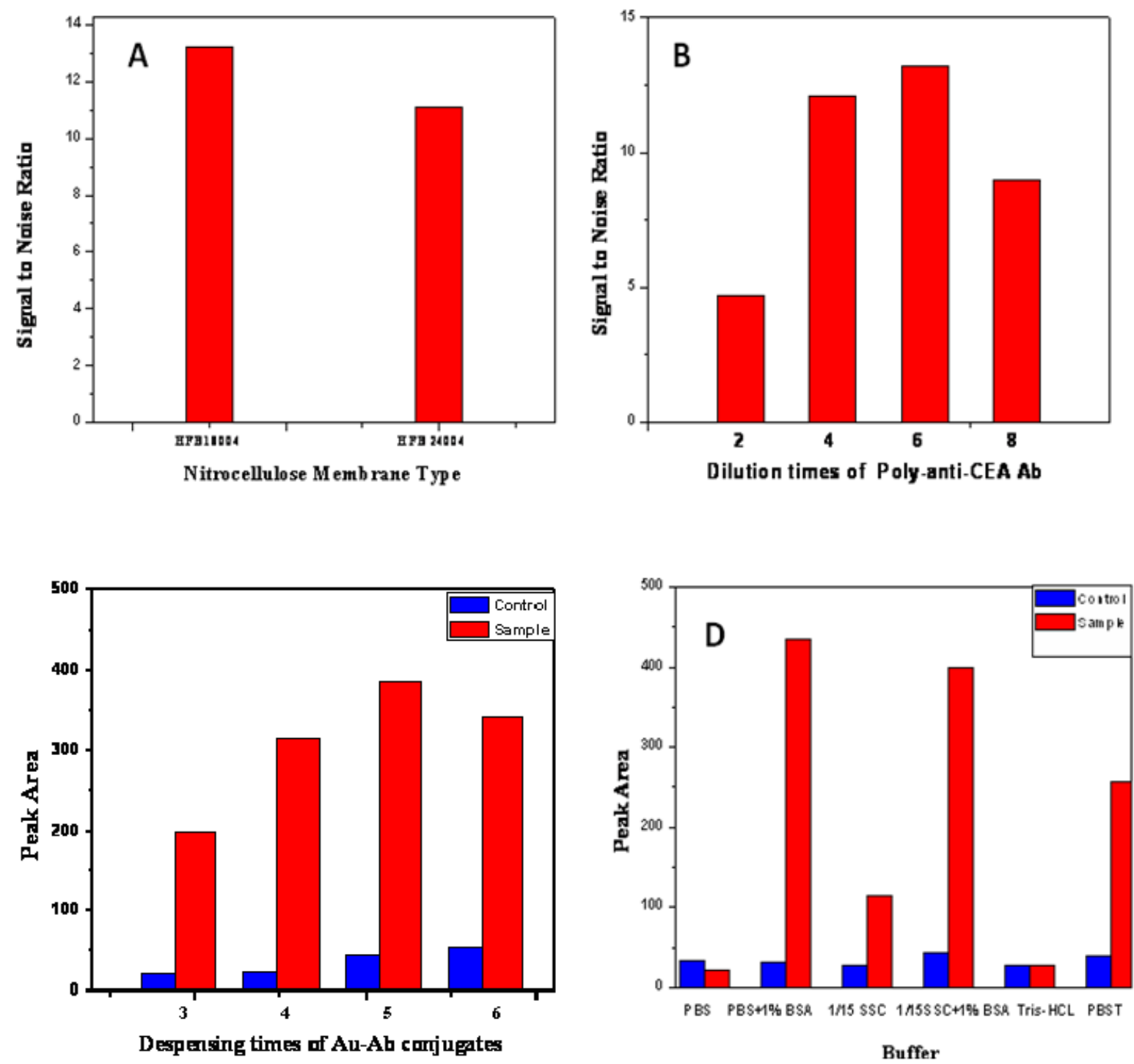

Figure 4. (A) Effect of nitrocellulose membrane sources on the signal to noise ratio of the biosensor; (B) Effect of the concentration of poly-anti-CEA Ab used for preparing test zone; (C) Effect of the amount of Au-NP-anti-CEA conjugates on the conjugate pad; (D) Effect of running buffer components on the response of the biosensor. The concentration of CEA in the sample solution is $200 \mathrm{ng} \mathrm{mL}^{-1}$. Assay time: $10 \mathrm{~min}$.

The response of the biosensor is relevant to the amount of poly-anti-CEA Ab immobilized on the test zone. The concentration of poly-anti-CEA $\mathrm{Ab}$ from commercial vendor is $2.4 \mathrm{mg} / \mathrm{mL}$, so the $\mathrm{Ab}$ solution with different dilution times was used to prepare the test zone of strip biosensor. One can see from the Figure 4(B), the $\mathrm{S} / \mathrm{N}$ ratio of the biosensor increased with the increasing of dilution times from 2 to 6 times, further dilution led to the decrease of $\mathrm{S} / \mathrm{N}$ ratio. There are two factors 
resulted the low $\mathrm{S} / \mathrm{N}$ ratio at high concentration of poly-anti-CEA Ab on the test zone: (1) The high concentration of $\mathrm{Ab}$ in test zone caused nonspecific adsorption of the conjugates in the test zone, resulting a high background signal; (2) The excess amount of $\mathrm{Ab}$ on the test zone may increase the stereo-hindrance effect which may decreasing the efficiency of immunoreactions.

Another factor taken into account for the assay is the amount of Au-NP-anti-CEA conjugates on the conjugate pad. The intensities of the produced red bands in the test zone and control zone depend on the amount of Au-NP-anti-CEA conjugates captured on the zones, which in turn corresponds to the amount of conjugates on the conjugate pad. In our study, the amount of the conjugates on the pad was controlled by the dispensing volumes of the conjugate solution, which were performed by dispensing the conjugate solution with various cycles on the conjugate pad. Figure 4 (C) presents the histogram of the recorded intensity of test line for $200 \mathrm{ng}$

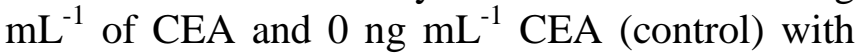
the different amount of Au-NP-anti-CEA conjugate loaded conjugate pads. As one can see, the responses of the biosensor for both sample and control increased with the increasing of dispensing times, however the maximum $\mathrm{S} / \mathrm{N}$ was obtained with 4 dispensing times on the conjugate pad. Therefore, four dispensing times was selected as the optimal dispensing time for most of the experiments.

The components of running buffer used in this study affected the response of the biosensor greatly. Appropriate buffer would minimize the nonspecific adsorption, increase the sensitivity and reproducibility of the biosensor. Different kinds of buffers were used in this study: (1) PBS, (2) PBS + 1\% BSA, (3) $1 / 15$ SSC, (4) $1 / 15 \mathrm{SSC}+1 \%$ BSA, (5) Tris- $\mathrm{HCl}$ (6) PBST (PBS + 0.25\% Tween 20). Figure 4 (D) presents the histogram of the biosensor response with different running buffers. One can see the buffer consisting of PBS $+1 \%$ BSA exhibited the best performance. So PBS + $1 \%$ BSA buffer was used for the following experiments.

Analytical Performance. Under optimal experimental conditions, we examined the performance of the biosensor with different concentrations of CEA in pure buffer solutions.
Quantitative detections were performed by recording the optical intensities of the test lines with portable strip reader. Well-defined peaks were observed and peak areas were increasing with the increase of CEA concentration. The resulting calibration plot (Figure 5) is linear over the $10-200 \mathrm{ng} \mathrm{mL}^{-1}$ range and is suitable for quantitative work. The linear equation is $\mathrm{Pa}=$ $1.32 \mathrm{c}+33.12\left(\mathrm{~Pa}\right.$ : peak area; $\left.\mathrm{c}: \mathrm{ng} \mathrm{mL}^{-1}\right)$ with $\mathrm{R}^{2}$ $=0.996$. The detection limit of $5 \mathrm{ng} \mathrm{mL}^{-1}$ (based on the response of $10 \mathrm{ng} \mathrm{mL} \mathrm{m}^{-1} \mathrm{CEA}$, inset) was estimated in connection with the 10-min assay time. Reproducibility of the biosensor was determined by a series of measurements of $100 \mathrm{ng}$ $\mathrm{mL}^{-1} \quad \mathrm{CEA}$ with six biosensors, yielded reproducible signals with an RSD of $8.9 \%$ (data not shown).

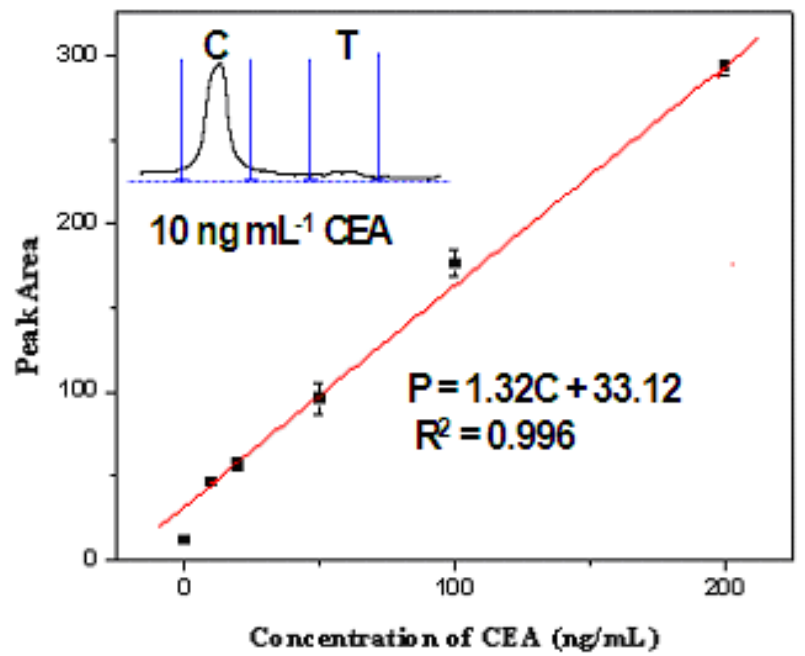

Figure 5. Calibration curve of the test strip with different concentrations of CEA in $0.01 \mathrm{M}$ PBS + $1 \%$ BSA buffer. Assay time: $10 \mathrm{~min}$; dispensing times of Au-anti-CEA conjugates on the conjugate pad: 2; dilution times of poly-anti-CEA $\mathrm{Ab}$ for preparing test line: 6 . $0.01 \mathrm{M} \mathrm{PBS}+1 \% \mathrm{BSA}$ solution as running buffer. Also shown (inset) is the optical response of $10 \mathrm{ng} \mathrm{mL}-1 \mathrm{CEA}$ on the strip biosensor.

Determination of CEA in Human Plasma. To demonstrate the feasibility of the biosensor for the quantitative detection of CEA, the device was then applied to detect CEA in human plasma sample. The sample solutions with different concentrations of CEA were prepared by spiking standard CEA into human serum. The serum 
sample without addition of standard CEA served as a control. The resulting plot of the peak areas versus CEA concentrations is linear over the 5 $500 \mathrm{ng} \mathrm{mL}^{-1}$ range with a detection limit of $5 \mathrm{ng}$ $\mathrm{mL}^{-1}$ (based on $\mathrm{S} / \mathrm{N}=3$ ). Although the detection limit of the biosensor is higher than that of commercial CEA ELISA kit $\left(0.64 \mathrm{ng} \mathrm{mL}^{-1}\right.$, BIOQUANT INC, SAN DIEGO, CA), the total assay time per sample with the QISB is $10 \mathrm{~min}$, which is much shorter than that of the commercial ELISA kit $(1.5 \mathrm{~h})$. Good performance of the CEA test strip in human plasma demonstrates the promise for further clinical applications.

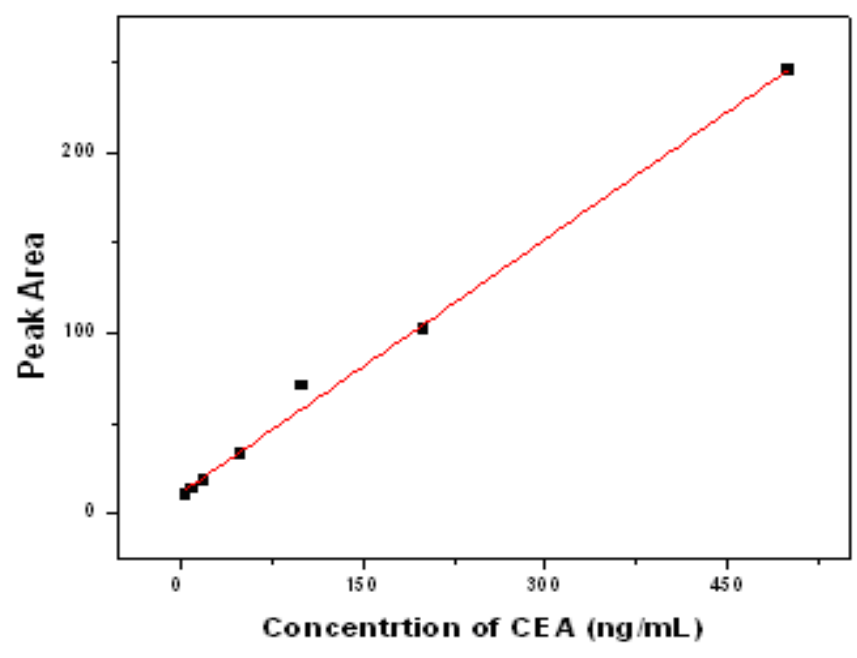

Figure 6. Calibration curve of the test strip with mixture of CEA antigen and plasma samples. $120 \mu \mathrm{L}$ of plasma containing different amount of CEA antigen was applied to the strip biosensor. Other conditions, same as in Figure 5.

\section{Conclusions}

We have successfully developed a quantitative immunochromatographic strip biosensor coupling a portable strip reader for rapid, low-cost and sensitive quantitative detection of CEA. Under optimal conditions, the response of the test strip is linear in the range of $10-200 \mathrm{ng}$ $\mathrm{mL}^{-1}$ with a detection limit of $5 \mathrm{ng} \mathrm{mL} \mathrm{m}^{-1}$ in association with a 10-min assay time. Moreover, the biosensor has been successfully applied for the detection of CEA in human plasma. Coupled with the portable strip reader, this strip biosensor shows great promise for point-of-care or in-field detection of protein biomarkers. The detection limit of the QISB is higher than that of the commercial CEA ELISA kit, future work will aim at to improve the sensitivity of the QISB by developing enzyme and gold nanoparticle dual labels based QISB, and to detect multiple protein biomarkers simultaneously with a QISB array.

\section{Acknowledgements}

This publication was made possible by $\mathrm{NIH}$ Grant Number 2P20 RR015566 from the National Center for Research Resources. Its contents are solely the responsibility of the authors and do not necessarily represent the official views of the NIH. G. Liu acknowledges the financial support from North Dakota Experimental Program to Stimulate Competitive Research (EPSCoR) and the new faculty startup funds of North Dakota State University. S. Wang acknowledges the financial support from the National Natural Science Foundation of China (No. 20575017).

\section{References}

[1] Gold P., Freedman S.O. J. Exp. Med. 1965, 121, 439-462.

[2] Jezersek B., Cervek J., Rudolf Z., Novaković S. Cancer Lett. 1996, 110, 137-144.

[3] Kleisbauer J.P., Castelnau 0., Thomas P., Ramirez J., Lanteaume A., Roux F. Lung Cancer 1996, 15, 148.

[4] Sahin B.; Paydak V.; Paydas S. Eur. J. Cancer 1996, 32, S24.

[5] Hammarstrom S., Shively J.E., Paxton R.J., Beatty B.G., Larsson A., Ghosh R., Bormer O., Buchegger F., Mach J.P, Burtin P. Cancer Res. 1989, 49, 4852-4858.

[6] Ford C.H.J., Osborne P.A., Rego B.G., Mathew A. Int. J. Cancer 2001, 92, 851-855.

[7] Duffy M.J., van Dalen A., Haglund C., Hansson L., Klapdor R., Lamerz R., Nilsson O., Sturgeon C., Topolcan O. Eur. J. Cancer 2003, 39, 718-727.

[8] Moro D., Villemain D., Vuillez J.P., Brambilla C. Lung Cancer 1995, 13, 169-176.

[9] Iwazawa T., Kanoh T., Matsui S., Monden T. Lung Cancer 2000,29, 254. 
[10] Kazuya K., Yoshihiro K., Tsunekazu K., Takehiko T., Masashi T., Junko H., Yoshinori M., Kenji Y., Ichiro N. Gynecol Obstet Invest 1999, 47, 52-57.

[11] Thompson D. M. P., Krupey, J., Freedman, S., Gold, P. Proc. Nat. A cad. Sci. 1969, 64, 161167.

[12] Szturmowicz M., Tomkowski W., Fijalkowska A., Sakowicz A., Filipecki S. Eur. J. Cancer 1995, 31, S264.

[13] Villena V., Lopez-Encuentra A., EchaveSustaeta J., Martin-Escribano P., Ortuno-de-Solo B., Estenoz-Alfaro J. Cancer 1996, 78, 736.

[14] Yang H., Zhu Q., Li D., Lin P., Ding M., Xu J. Fresenius J. Anal. Chem. 2001, 370, 88-91.

[15] Yuan J., Wang G., Majima K., Matsumoto K. Anal. Chem. 2001, 73, 1869-1876.

[16] Blackburn G.F., Shah H.P., Kenten J.H., Leland J., Kamin R.A., Link J. Peterman J., Powell M.J., Shah A., Talley D.B. Clin. Chem. 1991, 37, 1471-1664.

[17] Alumanda M.D.R., Minoru K. Anal. Chim. Acta 1995, 312, 85-94.

[18] Aguilar Z.P., Vandaveer W.R., Frisch I. Anal. Chem. 2002, 74, 3321-3329.

[19] Dai Z., Yan F., Yu H., Hu X., Ju H. J. Immunol. Methods 2004, 287, 13-20.

[20] Li X., Yuan R., Chai Y., Zhang L., Zhuo Y., Zhang Y. J. Biotechnol. 2006, 123, 356-366.

[21] Shi Y., Yuan R., Chai Y., He X. Electrochim. Acta 2007, 52, 3518-3524.

[22] Limbut W., Kanatharana P., Mattiasson B., Asawatreratanakul P., Thavarungkul P. Anal. Chim. Acta, 2006, 561, 55-61.
[23] Zhu Q., Chai Y., Yuan R., Wang N., Li X. Chem.Lett. 2005, 34, 1682-1683.

[24] Cui G., Kim S.J., Choi S.H., Nam H., Cha G.S. Anal. Chem. 2000, 72, 1925-1929.

[25] Glynou, K. Ioannou, P. C. Christopoulos, T. K. Syriopoulou, V. Anal. Chem. 2003, 75, 41554160.

[26] Jin, Y., Jang, J.W., Han, C.H., Lee, M.H. J. Agric. Food Chem. 2005, 53, 7639-7643.

[27] Zhu Y., Li L., Wang Z., Chen Y., Zhao Z., Zhu L., Wu X., Wan Y., He F., Shen J. J. Agric. Food Chem. 2008, 56, 5469-5474.

[28] Fernandez-Sanchez, C., McNeil, C.J., Rawson, K., Nilsson, O., Leung, H.Y., Gnanapragasam V. J. Immunol. Methods 2005, 307, 1-12.

[29] Kim, Y.M., Oh, S.W., Jeong, S.Y., Pyo, D.J., Choi, E.Y. Environ. Sci. Technol. 2003, 37, 18991904.

[30] Liu G., Lin Y., Wang J., Wu H., Wai C. M., and Lin Y. Anal. Chem, 2007, 79, 7644-7653.

[31] Lin, Y.; Wang, J.; Liu, G.; Wu, H.; Wai, C. M.; Lin, Y. Biosensors and Bioelectronics, 2008, 23, 1659-1665.

[32] Mao, X., Baloda, M., Gurung, A. S., Lin, Y., Liu, G. Electrochem.Commun. 2008, 10, 1636-1640.

[33] Grabar, K. C.; Freeman, R. G.; Hommer, M. B.; Natan, M. J. Anal.Chem. 1995, 67, 735-743.

[34] Frens, G. Nat. Phys. Sci. 1973, 241, 20-22. 